\title{
Naja naja karachiensis Envenomation: Biochemical Parameters for Cardiac, Liver, and Renal Damage along with Their Neutralization by Medicinal Plants
}

\author{
Muhammad Hassham Hassan Bin Asad, ${ }^{1}$ Ghulam Murtaza, \\ Muhammad Ubaid, ${ }^{1}$ Durr-e-Sabih, ${ }^{2}$ Ashif Sajjad, ${ }^{3}$ Rubada Mehmood, ${ }^{2}$ \\ Qaisar Mahmood, ${ }^{4}$ Muhammad Muzzmil Ansari, ${ }^{5}$ Sabiha Karim, \\ Zahid Mehmood, ${ }^{3}$ and Izhar Hussain ${ }^{1}$ \\ ${ }^{1}$ Department of Pharmacy, COMSATS Institute of Information Technology, Abbottabad 22060, Pakistan \\ ${ }^{2}$ Multan Institute of Nuclear Medicine and Radiotherapy (MINAR), 377, Nishtar Hospital, Multan 60000, Pakistan \\ ${ }^{3}$ Institute of Biochemistry, University of Balochistan, Quetta 87300, Pakistan \\ ${ }^{4}$ Department of Environmental Sciences, COMSATS Institute of Information Technology, Abbottabad 22060, Pakistan \\ ${ }^{5}$ Roba-al-Safwa Pharmacy, Alsafwa Hospital 67, Makkah, Saudi Arabia \\ ${ }^{6}$ University College of Pharmacy, University of the Punjab, Lahore 54000, Pakistan
}

Correspondence should be addressed to Ghulam Murtaza; gmdogar356@gmail.com and Izhar Hussain; izharhussain@ciit.net.pk

Received 17 January 2014; Accepted 27 March 2014; Published 27 April 2014

Academic Editor: Andrei Surguchov

Copyright (C) 2014 Muhammad Hassham Hassan Bin Asad et al. This is an open access article distributed under the Creative Commons Attribution License, which permits unrestricted use, distribution, and reproduction in any medium, provided the original work is properly cited.

\begin{abstract}
Naja naja karachiensis envenomation was found to hit more drastically heart, liver, and kidneys. $400 \mu \mathrm{g} / \mathrm{kg}$ of venom-raised moderate serum levels of ALT $(72 \pm 4.70 \mathrm{U} / \mathrm{L}, 0.1>P>0.05)$, AST $(157 \pm 24.24 \mathrm{U} / \mathrm{L}, 0.1>P>0.05)$, urea $(42 \pm 3.08 \mathrm{mg} / \mathrm{dL}$, $0.05>P>0.02)$, creatinine $(1.74 \pm 0.03 \mathrm{mg} / \mathrm{dL}, 0.01>P>0.001)$, CK-MB $(21 \pm 1.5 \mathrm{U} / \mathrm{L}, 0.05>P>0.02)$, and LDH $(2064 \pm 15.98 \mathrm{U} / \mathrm{L}, P<0.001)$ were injected in experimental rabbits. However, lethality was enhanced with $800 \mu \mathrm{g} / \mathrm{kg}$ of venom in terms of significant release of ALT $(86 \pm 5.0 \mathrm{U} / \mathrm{L}, 0.05>P>0.02)$, AST $(251 \pm 18.2 \mathrm{U} / \mathrm{L}, 0.01>P>0.001)$, urea $(57.6 \pm 3.84 \mathrm{mg} / \mathrm{dL}$, $0.02>P>0.01)$, creatinine $(2.1 \pm 0.10 \mathrm{mg} / \mathrm{dL}, 0.02>P>0.01)$, CK-MB $(77 \pm 11.22 \mathrm{U} / \mathrm{L}, 0.05>P>0.02)$, and $\mathrm{LDH}$ $(2562 \pm 25.14 \mathrm{U} / \mathrm{L}, P \ll 0.001)$. Among twenty-eight tested medicinal plant extracts, only Stenolobium stans (L.) Seem was found the best antivenom $(P>0.5)$ compared to the efficacy of standard antidote $(\mathrm{ALT}=52.5 \pm 3.51 \mathrm{U} / \mathrm{L}, \mathrm{AST}=69.5 \pm 18.55 \mathrm{U} / \mathrm{L}$, urea $=31.5 \pm 0.50 \mathrm{mg} / \mathrm{dL}$, creatinine $=1.08 \pm 0.02 \mathrm{mg} / \mathrm{dL}, \mathrm{CK}-\mathrm{MB}=09 \pm 0.85 \mathrm{U} / \mathrm{L}$, and $\mathrm{LDH}=763 \pm 6.01 \mathrm{U} / \mathrm{L}$ ). Other plant extracts were proved less beneficial and partly neutralized the toxicities posed by cobra venom. However, it is essential in future to isolate and characterize bioactive compound(s) from Stenolobium stans (L.) Seem extract to overcome the complications of snake bite.
\end{abstract}

\section{Introduction}

One of the animals which represent Pakistan and India throughout the world is cobra; in particular it is rearing out of a wicker basket and dancing to the sound of turban-wearing snake charmer music. In fact, they are deadly venomous among all species of the snakes due to high rate of mortality which strongly reasoned for their notoriety [1]. At the end of the nineteenth century, all cobra populations have been merged into single species naja (formerly known as naia); however, subsequently ten subspecies of Naja naja were identified [1]. Later on, Deraniyagala classified Naja naja (India) into several subspecies and considered Naja naja karachiensis one of them widely distributed in Southern Punjab province of Pakistan [2]. They are extremely toxic due to their severe side effects in the victims. Major signs and symptoms of cobra envenomation are edema, necrosis, pain, respiratory paralysis, vomiting, headache, hypotension, cardiac arrest, coagulopathies (elevated PT, aPTT, and TT), bleeding wounds, hematuria, mucus discharge, proteinuria, 
and increased creatinine and urea levels along with altered consciousness $[3,4]$.

Indeed snake venom is a complex mixture of various sizes of polypeptides $[5,6](<1.5 \mathrm{kDa}, 5$ to $10 \mathrm{kDa}$, and 10 to $150 \mathrm{kDa}$ ), carbohydrates, lipids, metal ions, organic molecules, purines, and amines $[7,8]$. Snake bite envenomation has been attributed to pose drastic changes in the physiology of the victims that could be accessed clinically by measuring various biochemical parameters. Among toxic components, phospholipases $\mathrm{A}_{2}$ (cell destroying enzymes cause edema, lipolytic or myolytic) $[9,10]$, phosphodiesterases (nucleic acid degrading enzyme causes hypotension/shock) [11, 12], $5^{\prime}$ nucleotidases (digest the sugar moities causing the delay in coagulation) [13], alkaline phosphatases (hydrolyze phosphate esters nonspecifically cause multiple toxicities via adenosine generation) [14], hyaluronidases (spreading factor causes digestion, necrosis, and leakage of blood vessels) [15], L-amino oxidases (deamination leads to cell damage/apoptosis) [16], and proteases (hydrolyze various proteins) [17] are included.

Laboratory animals, particularly rabbits, have been selected previously to monitor various biochemical changes related to liver, heart, and renal toxicities [18, 19]. Aspartate aminotransferase (GOT/AST) and alanine aminotransferase (GPT/ALT) are surrogate markers for liver toxicities while creatine kinase isoenzyme (CK-MB) is used exclusively to access cardiac tissue damage [20,21]. Elevated creatinine and urea levels are indicators of $75 \%$ renal damage [20], whereas high level of lactate dehydrogenase (LDH) implies general toxicity related to the liver, heart, kidneys, and skeleton muscles [22].

Diverse immunological properties of different cobras have fascinated protein chemists and other researchers to carry out efforts for the development of new antidotes. Due to this reason, for the first time, venom from Naja naja karachiensis is selected to evaluate its toxicity in vivo and to search out effective as well as cheap treatment from folk herbal remedies. Pakistan is a hub of medicinal plants where mostly rural communities rely on natural herbs to treat their problems of snake bite $[6,23]$. It is therefore necessary to evaluate scientifically their folklore claims as anti-snake venom in traditional system of medicine. Due to this reason, various medicinal plants (Albizia lebbeck (L.) Benth., Allium cepa L., Allium sativum L., Althaea officinalis L., Bauhinia variegata L., Brassica nigra (L.) W. D. J. Koch, Calotropis procera (Aiton) W. T. Aiton, Cedrus deodara (Roxb. ex D. Don) G. Don, Citrullus colocynthis (L.) Schrad, Citrus limon (L.) Burm. f., Cuminum cyminum L., Enicostema hyssopifolium (Willd.) I. Verd, Fagonia cretica L., Leucas capitata Desf., Matthiola incana (L.) W. T. Aiton, Momordica charantia L., Nerium indicum Mill, Ocimum sanctum L., Pinus roxburghii Sarg, Pistacia integerrima J. L. Stewart, Psoralea corylifolia L., Rhazya stricta Decne, Rubia cordifolia L., Sapindus mukorossi Gaertn, Solanum xanthocarpum Schard and Wendle, Stenolobium stans (L.) Seem, Terminalia arjuna (Roxb. ex DC.) Wight and Arn, Trichodesma indicum (L.) Sm, and Zingiber officinale Roscoe) were collected to test their potentials against toxicities induced by Naja naja karachiensis envenomation (Table 1).

\section{Material and Methods}

2.1. Collection and Milking of Snake Venom. Black Pakistani cobra snakes (Naja naja karachiensis) were collected from Cholistan desert located in southern Punjab province of Pakistan. After collection, they were properly identified by a zoologist. Venom from Naja naja karachiensis was collected by pressing their glands below their eyes in low light environment. After collection, it was lyophilized and stored in light resistant container at $2^{\circ} \mathrm{C}$ to $8^{\circ} \mathrm{C}$. For further experiments, venom was used in terms of its dry weight [6].

2.2. Collection and Preparation of Plant Extracts. Medicinal plants were collected from different areas in Pakistan. After collection, they were duly identified by expert botanist (Professor Dr. Altaf Ahmad Dasti) and voucher specimens were deposited in the herbarium of the Institute of Pure and Applied Biology, Bahauddin Zakariya University, Multan, Pakistan. Complete description about evaluated medicinal plants is summarized in Table 1. One kilogram chopped plant material was soaked in $5 \mathrm{~L}$ of methanol as solvent in extraction bottles. The homogenates were kept for a period of a month at optimum temperature. After filtration, filtrate was evaporated by using water bath and extracts were weighed and stored for further experimentation [24].

2.3. Experimental Animals. Seventy-one healthy growing male rabbits $(1 \pm 0.5 \mathrm{~kg})$ were selected for this study after getting permission from local ethical committee. Animals were acclimatized for a week in the laboratory by maintaining their standards for chow, water, and light. Subsequently, they were divided into various groups in different cages such that experimental rabbits in group I were used for baseline measurements of various biochemical parameters. Animals belonging to groups II and III were evaluated for different doses of cobra venom while group IV was served as control. Group V was redivided into twenty-nine groups such that each subgroup (V/1-V/29) was assigned to a single medicinal plant extract [25].

2.4. In Vivo Antivenom Activity of Medicinal Plant Extracts. Venom from Naja naja karachiensis $(400 \mu \mathrm{g} / \mathrm{kg}$ and $800 \mu \mathrm{g} / \mathrm{kg}$ ) was injected subcutaneously to evaluate its toxicity to the heart, liver, and kidneys. Before injection of venom, experimental animals were anaesthetized by administration of ketamine $(50 \mathrm{mg} / \mathrm{kg})$. To determine antivenom activity of various plants' extract, they $(100 \mathrm{mg} / \mathrm{kg})$ were incubated (at $37^{\circ} \mathrm{C}$ for 30 minutes) with fixed amount of venom $(800 \mu \mathrm{g} / \mathrm{kg})$ before injection while saline was used as control [26-28].

2.5. Biochemical Assays. For serum analysis of various biochemical parameters, blood was collected from marginal ear artery by the use of hypodermic syringe needle after 3 hours of envenomation [25]. Separated serum was tested for alanine aminotransferase (GPT/ALT), aspartate aminotransferase (GOT/AST), urea, creatinine, creatine kinase isoenzyme MB (CK-MB), and lactate dehydrogenase $(\mathrm{LDH})$ by the use of 


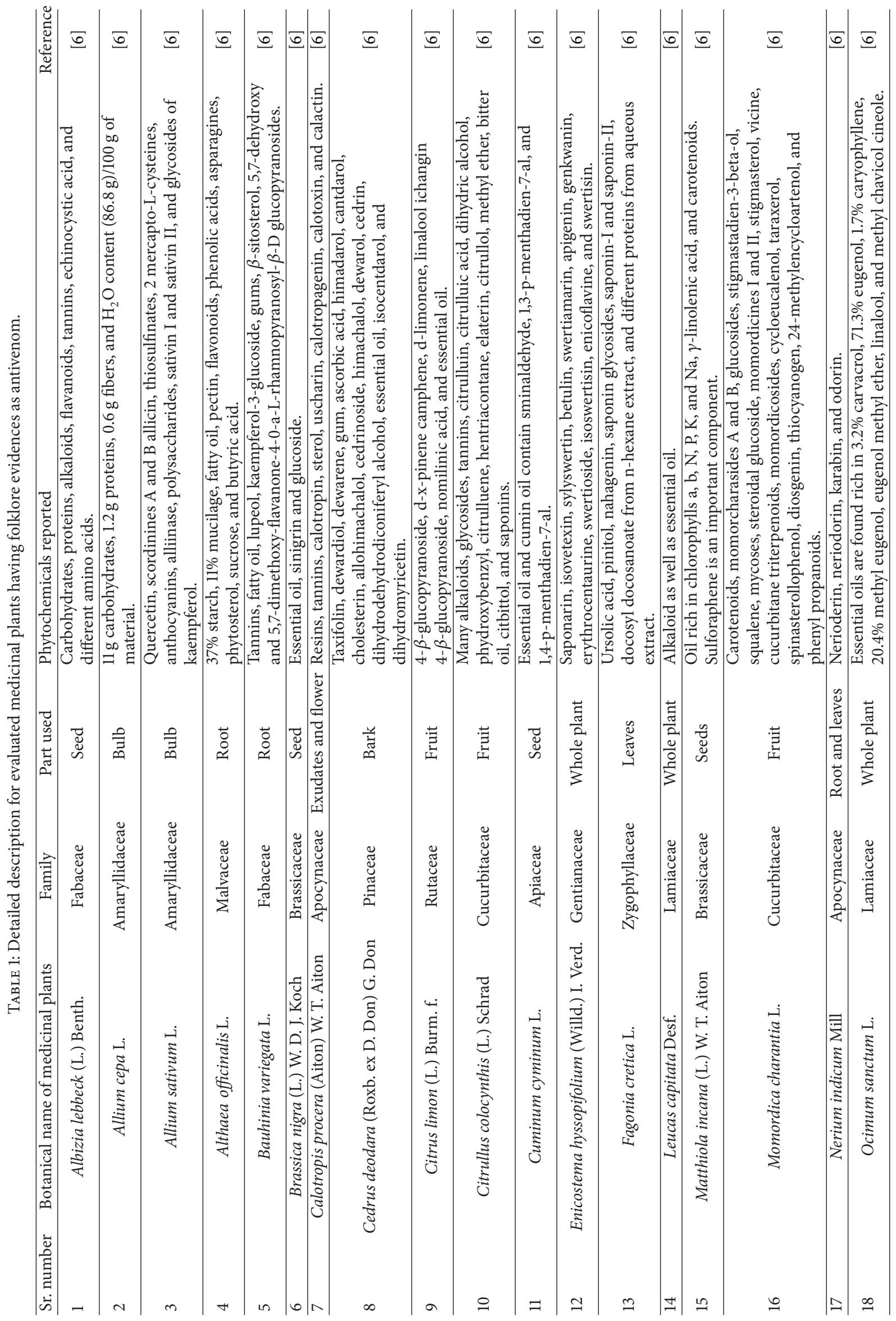




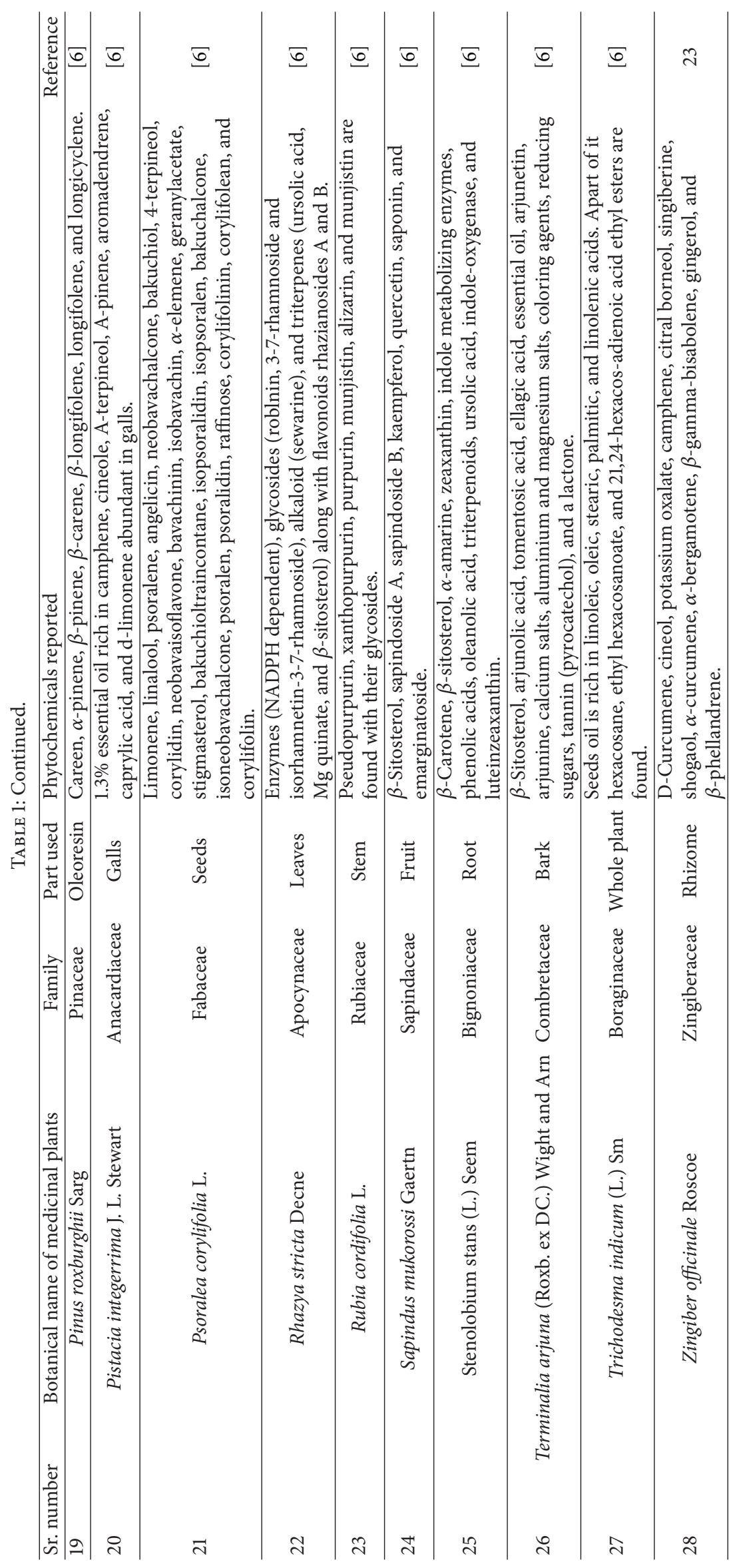




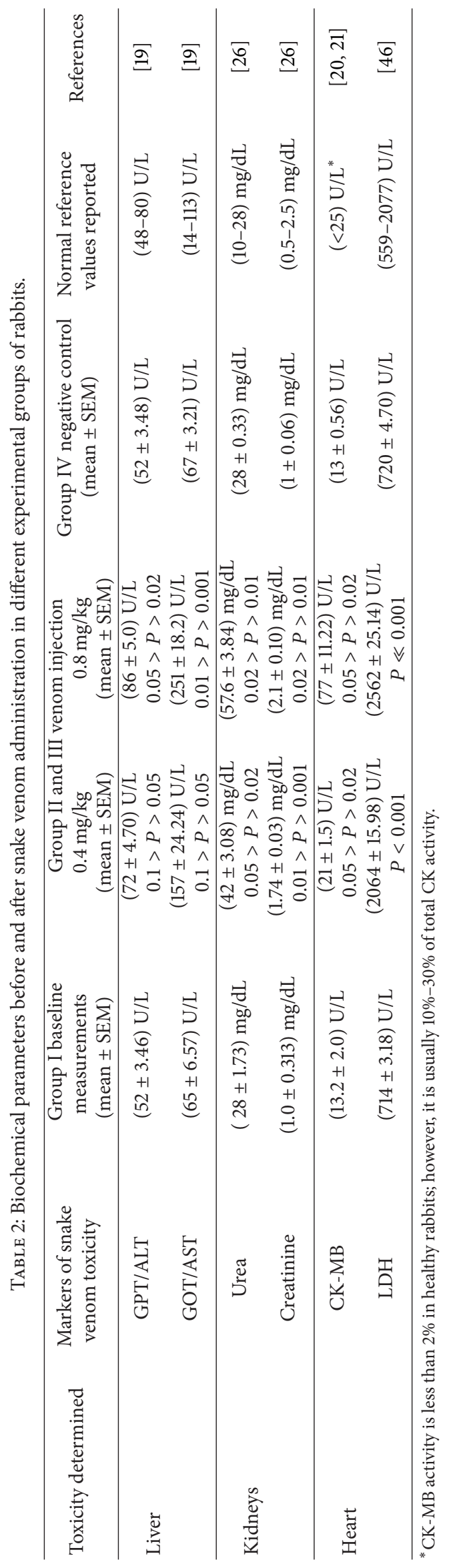




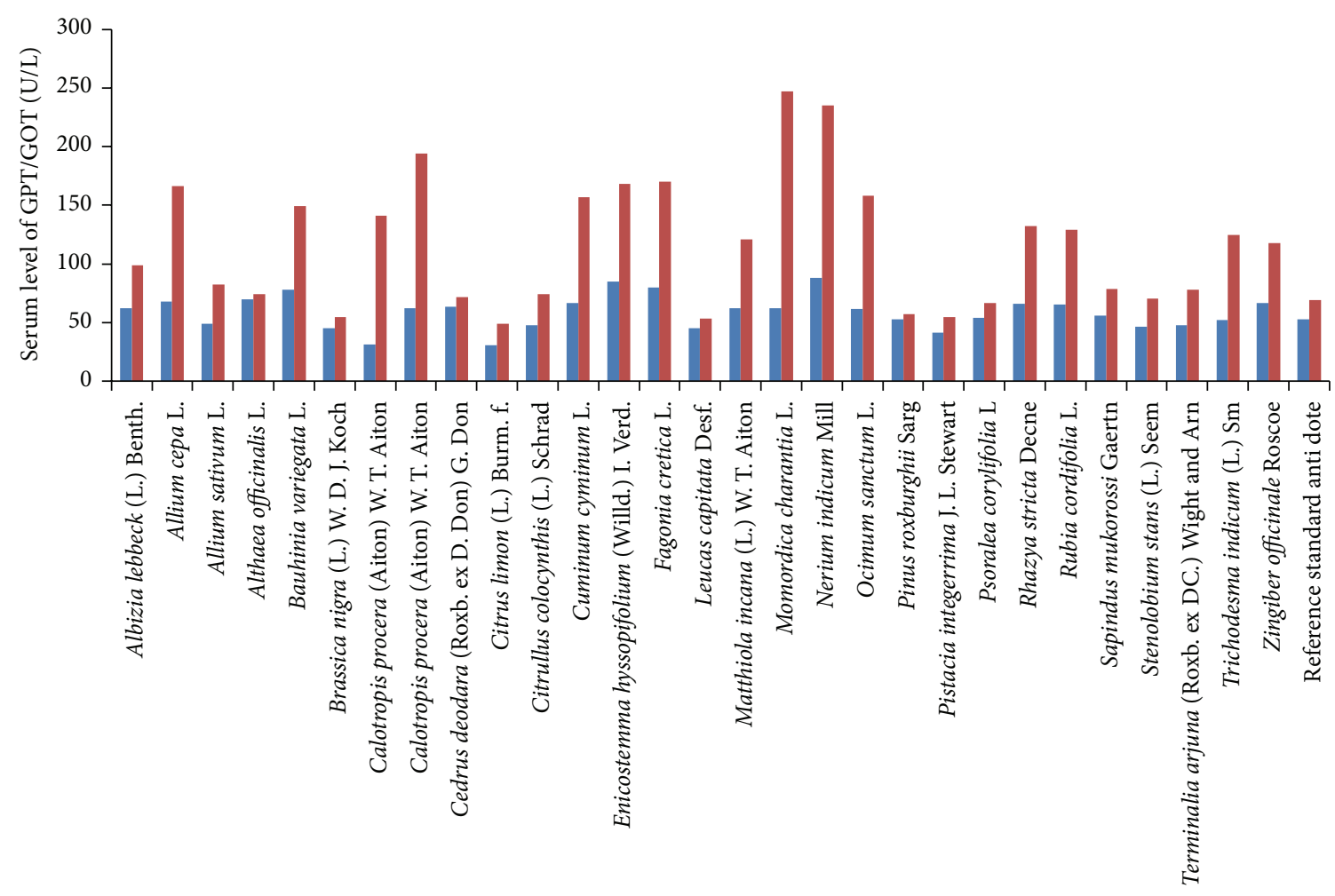

Various antidotes used $(100 \mathrm{mg} / \mathrm{kg})$

$$
\begin{aligned}
& \text { GPT level } \\
& \text { GOT level }
\end{aligned}
$$

FIGURE 1: Comparison of various medicinal plant extracts with reference standard antidote in neutralization of increased GPT and GOT serum level posed by Naja naja karachiensis venom in rabbits.

kits (manufactured by Merck) according to the DGKC and IFCC method on the Selectra Junior (Vital Scientific B.V, The Netherlands) [26].

2.6. Statistical Analysis. All numerical values were expressed as mean (3 replicates) \pm standard error of mean (SEM). They were calculated by the use of Microsoft Excel 2007 and Student's $t$-test was applied to compare the efficacy of evaluated samples with standard antidote (reference standard).

\section{Results}

Venom from Naja naja karachiensis was proved to cause severe complications in dose-dependent manner. Venom at the dose of $400 \mu \mathrm{g} / \mathrm{kg}$ led to release of moderate serum levels of ALT $(72 \pm 4.70 \mathrm{U} / \mathrm{L}, 0.1>P>0.05)$, AST $(157 \pm 24.24 \mathrm{U} / \mathrm{L}$, $0.1>P>0.05)$, urea $(42 \pm 3.08 \mathrm{mg} / \mathrm{dL}, 0.05>P>0.02)$, creatinine $(1.74 \pm 0.03 \mathrm{mg} / \mathrm{dL}, 0.01>P>0.001)$, CK-MB $(21 \pm 1.5 \mathrm{U} / \mathrm{L}, 0.05>P>0.02)$, and LDH $(2064 \pm 15.98 \mathrm{U} / \mathrm{L}$, $P<0.001)$ and thus indicated toxicities to the heart, liver, and kidneys. At $800 \mu \mathrm{g} / \mathrm{kg}$ of cobra venom, severe tissue damage was observed in terms of significant release of ALT (86 \pm $5.0 \mathrm{U} / \mathrm{L}, 0.05>P>0.02)$, AST $(251 \pm 18.2 \mathrm{U} / \mathrm{L}, 0.01>P>$ $0.001)$, urea $(57.6 \pm 3.84 \mathrm{mg} / \mathrm{dL}, 0.02>P>0.01)$, creatinine
$(2.1 \pm 0.10 \mathrm{mg} / \mathrm{dL}, 0.02>P>0.01)$, CK-MB $(77 \pm 11.22 \mathrm{U} / \mathrm{L}$, $0.05>P>0.02)$, and LDH $(2562 \pm 25.14 \mathrm{U} / \mathrm{L}, P \ll 0.001)$. Complete detail about various biochemical parameters for baseline measurements different doses of cobra venom, and saline (as negative control) is summarized in Table 2.

To neutralize in vivo $800 \mu \mathrm{g} / \mathrm{kg}$ of cobra venom, twentyeight medicinal plant extracts were evaluated. It was noticed that extract of Stenolobium stans (L.) Seem was the best antidote $(P>0.5)$ compared to reference standard. Allium sativum L., Althaea officinalis L., Citrullus colocynthis (L.) Schrad, Leucas capitata Desf., Pinus roxburghii Sarg, Psoralea corylifolia L., Rubia cordifolia L., and Sapindus mukorossi Gaertn were found to be valuable plants to protect liver damage $(P>0.5)$ as standard antidote (ALT $=52.5 \pm$ $3.51 \mathrm{U} / \mathrm{L}$ and $\mathrm{AST}=69.5 \pm 18.55 \mathrm{U} / \mathrm{L})$; however, required ALT and AST levels were not observed with remaining plant extracts $(0.5>P>0.05)$ as shown in Table 3 and comparison is shown in Figure 1. Extracts of Leucas capitata Desf. and Althaea officinalis L. were observed to be helpful $(P>0.5)$ as reference standard (urea $=31.5 \pm 0.50 \mathrm{mg} / \mathrm{dL}$ and creatinine $=1.08 \pm 0.02 \mathrm{mg} / \mathrm{dL}$ ); nevertheless, remaining plants were found less valuable $(0.5>P>0.01)$ to minimize urea and creatinine levels to maintain kidney functions (Table 4 and Figure 2). When compared with standard antidote $(\mathrm{CK}-\mathrm{MB}=9.0 \pm 0.85 \mathrm{U} / \mathrm{L})$ eight medicinal plants 
TABLE 3: Hepatoprotective activity of various medicinal plant extracts on ALT and AST levels to neutralize snake bite envenomation in experimental rabbits.

\begin{tabular}{|c|c|c|c|c|c|c|}
\hline \multirow{2}{*}{ Sr. number } & \multirow{2}{*}{$\begin{array}{l}\text { Group V } \\
\text { (subgroup) }\end{array}$} & \multirow{2}{*}{ Name of evaluated sample } & \multicolumn{2}{|c|}{ GPT/ALT } & \multicolumn{2}{|c|}{ GOT/AST } \\
\hline & & & $\begin{array}{c}\mathrm{U} / \mathrm{L} \\
(\mathrm{mean} \pm \mathrm{SEM})\end{array}$ & $\begin{array}{l}P \text { value/ } \\
\text { comment }\end{array}$ & $\begin{array}{c}\mathrm{U} / \mathrm{L} \\
(\mathrm{mean} \pm \mathrm{SEM})\end{array}$ & $\begin{array}{l}P \text { value/ } \\
\text { comment }\end{array}$ \\
\hline 1 & $(\mathrm{~V} / 1)$ & Albizia lebbeck (L.) Benth. & $62 \pm 7.02$ & $0.5>P>0.1$ & $99 \pm 18.55$ & $0.5>P>0.1$ \\
\hline 2 & $(\mathrm{~V} / 2)$ & Allium cepa L. & $68 \pm 12.03$ & $0.5>P>0.1$ & $166.5 \pm 0.50$ & $0.5>P>0.1$ \\
\hline 3 & $(\mathrm{~V} / 3)$ & Allium sativum $\mathrm{L}$. & $49 \pm 5.01$ & $P>0.5$ & $82.5 \pm 18.55$ & $P>0.5$ \\
\hline 4 & $(\mathrm{~V} / 4)$ & Althaea officinalisL. & $70 \pm 20.05$ & $P>0.5$ & $74.5 \pm 14.54$ & $P>0.5$ \\
\hline 5 & $(\mathrm{~V} / 5)$ & Bauhinia variegata $\mathrm{L}$. & $78 \pm 4.04$ & $0.5>P>0.1$ & $149.5 \pm 19.9$ & $0.5>P>0.1$ \\
\hline 6 & $(\mathrm{~V} / 6)$ & Brassica nigra (L.) W. D. J. Koch & $45 \pm 0.00$ & $0.5>P>0.1$ & $55 \pm 1.00$ & $P>0.5$ \\
\hline 7 & $(\mathrm{~V} / 7 \mathrm{a})$ & $\begin{array}{l}\text { Calotropis procera (Aiton) W. T. } \\
\text { Aiton (exudates) }\end{array}$ & $31.5 \pm 0.5$ & $0.5>P>0.1$ & $141 \pm 1.01$ & $0.5>P>0.1$ \\
\hline 8 & $(\mathrm{~V} / 7 \mathrm{~b})$ & $\begin{array}{l}\text { Calotropis procera (Aiton) W. T. } \\
\text { Aiton (flowers) }\end{array}$ & $62.5 \pm 2.52$ & $0.5>P>0.1$ & $194 \pm 12.12$ & $0.5>P>0.1$ \\
\hline 9 & $(\mathrm{~V} / 8)$ & $\begin{array}{l}\text { Cedrus deodara (Roxb. ex D. } \\
\text { Don) G. Don }\end{array}$ & $63.3 \pm 4.14$ & $0.5>P>0.1$ & $72 \pm 2.02$ & $P>0.5$ \\
\hline 10 & $(\mathrm{~V} / 9)$ & Citrus limon (L.) Burm. f. & $30.5 \pm 13.63$ & $0.5>P>0.1$ & $49 \pm 24.24$ & $P>0.5$ \\
\hline 11 & $(\mathrm{~V} / 10)$ & Citrullus colocynthis (L.) Schrad & $47.5 \pm 5.55$ & $P>0.5$ & $74 \pm 9.09$ & $P>0.5$ \\
\hline 12 & $(\mathrm{~V} / 11)$ & Cuminum cyminum $\mathrm{L}$. & $67 \pm 16.16$ & $P>0.5$ & $157 \pm 14.14$ & $0.5>P>0.1$ \\
\hline 13 & $(\mathrm{~V} / 12)$ & $\begin{array}{l}\text { Enicostemma hyssopifolium } \\
\text { (Willd.) I. Verd. }\end{array}$ & $85.25 \pm 1.26$ & $\begin{array}{l}0.1>P> \\
\quad 0.05\end{array}$ & $168.5 \pm 7.57$ & $0.5>P>0.1$ \\
\hline 14 & $(\mathrm{~V} / 13)$ & Fagonia cretica L. & $80 \pm 7.82$ & $0.5>P>0.1$ & $170 \pm 13.13$ & $0.5>P>0.1$ \\
\hline 15 & $(\mathrm{~V} / 14)$ & Leucas capitata Desf. & $45.5 \pm 7.52$ & $P>0.5$ & $53.5 \pm 0.50$ & $P>0.5$ \\
\hline 16 & $(\mathrm{~V} / 15)$ & Matthiola incana (L.) W. T. Aiton & $62 \pm 8.08$ & $0.5>P>0.1$ & $121 \pm 5.05$ & $0.5>P>0.1$ \\
\hline 17 & $(\mathrm{~V} / 16)$ & Momordica charantia L. & $62.5 \pm 7.52$ & $0.5>P>0.1$ & $247 \pm 46.13$ & $0.5>P>0.1$ \\
\hline 18 & $(\mathrm{~V} / 17)$ & Nerium indicum Mill & $88 \pm 19.55$ & $0.5>P>0.1$ & $235 \pm 18.05$ & $\begin{array}{l}0.1>P> \\
\quad 0.05\end{array}$ \\
\hline 19 & $(\mathrm{~V} / 18)$ & Ocimum sanctum L. & $61.5 \pm 7.52$ & $0.5>P>0.1$ & $158 \pm 10.02$ & $0.5>P>0.1$ \\
\hline 20 & $(\mathrm{~V} / 19)$ & Pinus roxburghii Sarg & $53 \pm 9.02$ & $P>0.5$ & $57 \pm 13.03$ & $P>0.5$ \\
\hline 21 & $(\mathrm{~V} / 20)$ & Pistacia integerrima J. L. Stewart & $41.5 \pm 1.5$ & $0.5>P>0.1$ & $54.5 \pm 0.50$ & $P>0.5$ \\
\hline 22 & $(\mathrm{~V} / 21)$ & Psoralea corylifolia L. & $54 \pm 1.00$ & $P>0.5$ & $66.5 \pm 11.53$ & $P>0.5$ \\
\hline 23 & $(\mathrm{~V} / 22)$ & Rhazya stricta Decne & $66 \pm 24.24$ & $P>0.5$ & $132.5 \pm 12.62$ & $0.5>P>0.1$ \\
\hline 24 & $(\mathrm{~V} / 23)$ & Rubia cordifolia L. & $65.5 \pm 17.67$ & $P>0.5$ & $129 \pm 66.41$ & $P>0.5$ \\
\hline 25 & $(\mathrm{~V} / 24)$ & Sapindus mukorossi Gaertn & $56 \pm 6.01$ & $P>0.5$ & $78.5 \pm 13.54$ & $P>0.5$ \\
\hline 26 & $(\mathrm{~V} / 25)$ & Stenolobium stans (L.) Seem & $46.5 \pm 6.56$ & $P>0.5$ & $70.5 \pm 3.53$ & $P>0.5$ \\
\hline 27 & $(\mathrm{~V} / 26)$ & $\begin{array}{l}\text { Terminalia arjuna (Roxb. ex } \\
\text { DC.) Wight and Arn }\end{array}$ & $47.5 \pm 1.51$ & $0.5>P>0.1$ & $78 \pm 6.06$ & $P>0.5$ \\
\hline 28 & $(\mathrm{~V} / 27)$ & Trichodesma indicum (L.) Sm & $52 \pm 0.00$ & $P>0.5$ & $125 \pm 4.01$ & $0.5>P>0.1$ \\
\hline 29 & $(\mathrm{~V} / 28)$ & Zingiber officinale Roscoe & $66.5 \pm 32.59$ & $P>0.5$ & $117.5 \pm 9.52$ & $0.5>P>0.1$ \\
\hline 30 & $(\mathrm{~V} / 29)$ & $\begin{array}{l}\text { Reference standard antidote } \\
\text { (standard antisera } \\
\text { /immunoglobulin's) }\end{array}$ & $52.5 \pm 3.51$ & $\begin{array}{l}\text { Select to } \\
\text { compare }\end{array}$ & $69.5 \pm 18.55$ & $\begin{array}{l}\text { Select to } \\
\text { compare }\end{array}$ \\
\hline
\end{tabular}

(Allium cepa L., Althaea officinalis L., Bauhinia variegata L., Cedrus deodara (Roxb. ex D. Don) G. Don, Fagonia cretica L., Leucas capitata Desf., Momordica charantia L., and Ocimum sanctum L.) were found equally capable $(P>0.5)$ of normalizing high values of $\mathrm{CK}-\mathrm{MB}$; however, rest of all were proved less beneficial $(0.5>P>0.1)$. To combat highly raised values of LDH, Althaea officinalis L., Leucas capitata Desf., and Terminalia arjuna (Roxb. ex DC.) Wight and Arn were proved useful to some extent $(0.5>P>0.1)$ although remaining plant extracts could not be shortlisted $(0.1>P>$ $0.001)$ as standard antisera $(\mathrm{LDH}=763 \pm 6.01 \mathrm{U} / \mathrm{L})$. Overall detail about LDH is discussed in Table 5 and comparison is shown in Figure 3.

\section{Discussion}

Snake bite has been responsible for tens of thousands of deaths worldwide and numerous physical handicaps [29]. 
TABLE 4: Nephroprotective activity of different medicinal plant extracts on urea and creatinine levels to neutralize snake bite envenomation in experimental rabbits.

\begin{tabular}{|c|c|c|c|c|c|c|}
\hline \multirow{2}{*}{ Sr. number } & \multirow{2}{*}{$\begin{array}{l}\text { Group V } \\
\text { (subgroup) }\end{array}$} & \multirow{2}{*}{ Name of evaluated sample } & \multicolumn{2}{|c|}{ Urea } & \multicolumn{2}{|c|}{ Creatinine } \\
\hline & & & $\begin{array}{c}\mathrm{mg} / \mathrm{dL} \\
(\mathrm{mean} \pm \mathrm{SEM})\end{array}$ & $\begin{array}{l}P \text { value/ } \\
\text { comment }\end{array}$ & $\begin{array}{c}\mathrm{mg} / \mathrm{dL} \\
(\mathrm{mean} \pm \mathrm{SEM})\end{array}$ & $\begin{array}{l}P \text { value/ } \\
\text { comment }\end{array}$ \\
\hline 1 & $(\mathrm{~V} / 1)$ & Albizia lebbeck (L.) Benth. & $57 \pm 0.0$ & $0.02>P>0.01$ & $1.6 \pm 0.20$ & $0.5>P>0.1$ \\
\hline 2 & $(\mathrm{~V} / 2)$ & Allium cepa L. & $51 \pm 11.03$ & $0.5>P>0.1$ & $1.50 \pm 0.17$ & $0.5>P>0.1$ \\
\hline 3 & $(\mathrm{~V} / 3)$ & Allium sativum $\mathrm{L}$. & $50 \pm 4.01$ & $0.5>P>0.1$ & $1.30 \pm 0.06$ & $0.5>P>0.1$ \\
\hline 4 & $(\mathrm{~V} / 4)$ & Althaea officinalis L. & $26 \pm 5.05$ & $P>0.5$ & $1.18 \pm 0.24$ & $P>0.5$ \\
\hline 5 & $(\mathrm{~V} / 5)$ & Bauhinia variegata $\mathrm{L}$. & $50 \pm 12.6$ & $0.5>P>0.1$ & $1.36 \pm 0.24$ & $0.5>P>0.1$ \\
\hline 6 & $(\mathrm{~V} / 6)$ & Brassica nigra (L.) W. D. J. Koch & $22.5 \pm 4.51$ & $0.5>P>0.1$ & $1.07 \pm 0.11$ & $P>0.5$ \\
\hline 7 & $(\mathrm{~V} / 7 \mathrm{a})$ & $\begin{array}{l}\text { Calotropis procera (Aiton) W. T. } \\
\text { Aiton (exudates) }\end{array}$ & $42.5 \pm 2.52$ & $0.5>P>0.1$ & $1.30 \pm 0.01$ & $0.1>P>0.05$ \\
\hline 8 & $(\mathrm{~V} / 7 \mathrm{~b})$ & $\begin{array}{l}\text { Calotropis procera (Aiton) W. T. } \\
\text { Aiton (flowers) }\end{array}$ & $39 \pm 2.02$ & $0.5>P>0.1$ & $1.25 \pm 0.03$ & $0.5>P>0.1$ \\
\hline 9 & $(\mathrm{~V} / 8)$ & $\begin{array}{l}\text { Cedrus deodara (Roxb. ex D. } \\
\text { Don) G. Don }\end{array}$ & $44 \pm 4.04$ & $0.5>P>0.1$ & $1.44 \pm 0.07$ & $0.5>P>0.1$ \\
\hline 10 & $(\mathrm{~V} / 9)$ & Citrus limon (L.) Burm. f. & $39.5 \pm 2.52$ & $0.5>P>0.1$ & $1.40 \pm 0.03$ & $0.1>P>0.05$ \\
\hline 11 & $(\mathrm{~V} / 10)$ & Citrullus colocynthis (L.) Schrad & $49.5 \pm 3.53$ & $0.5>P>0.1$ & $1.52 \pm 0.15$ & $0.5>P>0.1$ \\
\hline 12 & $(\mathrm{~V} / 11)$ & Cuminum cyminum L. & $54 \pm 1.01$ & $0.05>P>0.02$ & $1.48 \pm 0.01$ & $0.05>P>0.02$ \\
\hline 13 & $(\mathrm{~V} / 12)$ & $\begin{array}{l}\text { Enicostemma hyssopifolium } \\
\text { (Willd.) I. Verd. }\end{array}$ & $39.5 \pm 4.54$ & $0.5>P>0.1$ & $1.35 \pm 0.05$ & $0.5>P>0.1$ \\
\hline 14 & $(\mathrm{~V} / 13)$ & Fagonia cretica L. & $43 \pm 2.27$ & $0.5>P>0.1$ & $1.23 \pm 0.13$ & $P<0.5$ \\
\hline 15 & $(\mathrm{~V} / 14)$ & Leucas capitata Desf. & $31.5 \pm 0.50$ & $P>0.5$ & $1.07 \pm 0.05$ & $P>0.5$ \\
\hline 16 & $(\mathrm{~V} / 15)$ & Matthiola incana (L.) W. T. Aiton & $43.5 \pm 3.53$ & $0.5>P>0.1$ & $1.44 \pm 0.14$ & $0.5>P>0.1$ \\
\hline 17 & $(\mathrm{~V} / 16)$ & Momordica charantia L. & $57 \pm 5.01$ & $0.5>P>0.1$ & $1.5 \pm 0.005$ & $0.05>P>0.02$ \\
\hline 18 & $(\mathrm{~V} / 17)$ & Nerium indicum Mill & $47 \pm 0.00$ & $P<0.05$ & $1.24 \pm 0.10$ & $0.5>P>0.1$ \\
\hline 19 & $(\mathrm{~V} / 18)$ & Ocimum sanctum L. & $49.5 \pm 7.52$ & $0.5>P>0.1$ & $1.5 \pm 0.005$ & $0.05>P>0.02$ \\
\hline 20 & $(\mathrm{~V} / 19)$ & Pinus roxburghii Sarg & $45 \pm 1.00$ & $P>0.05$ & $1.4 \pm 0.05$ & $0.5>P>0.1$ \\
\hline 21 & $(\mathrm{~V} / 20)$ & Pistacia integerrima J. L. Stewart & $66.5 \pm 0.50$ & $0.02>P>0.01$ & $1.68 \pm 0.10$ & $0.5>P>0.1$ \\
\hline 22 & $(\mathrm{~V} / 21)$ & Psoralea corylifolia $\mathrm{L}$. & $42.5 \pm 2.50$ & $0.5>P>0.1$ & $1.27 \pm 0.15$ & $0.5>P>0.1$ \\
\hline 23 & $(\mathrm{~V} / 22)$ & Rhazya stricta Decne & $53.5 \pm 3.53$ & $0.5>P>0.1$ & $1.67 \pm 0.005$ & $0.05>P>0.02$ \\
\hline 24 & $(\mathrm{~V} / 23)$ & Rubia cordifolia L. & $36 \pm 1.01$ & $0.5>P>0.1$ & $0.93 \pm 0.48$ & $P>0.5$ \\
\hline 25 & $(\mathrm{~V} / 24)$ & Sapindus mukorossi Gaertn & $44 \pm 1.00$ & $0.1>P>0.05$ & $1.43 \pm 0.12$ & $0.5>P>0.1$ \\
\hline 26 & $(\mathrm{~V} / 25)$ & Stenolobium stans (L.) Seem & $25.5 \pm 6.51$ & $P>0.5$ & $1.1 \pm 0.06$ & $P>0.5$ \\
\hline 27 & $(\mathrm{~V} / 26)$ & $\begin{array}{l}\text { Terminalia arjuna (Roxb. ex } \\
\text { DC.) Wight and Arn }\end{array}$ & $38.5 \pm 1.51$ & $0.5>P>0.1$ & $1.27 \pm 0.23$ & $P>0.5$ \\
\hline 28 & $(\mathrm{~V} / 27)$ & Trichodesma indicum (L.) Sm & $37 \pm 1.00$ & $0.5>P>0.1$ & $0.46 \pm 0.01$ & $0.05>P>0.02$ \\
\hline 29 & $(\mathrm{~V} / 28)$ & Zingiber officinale Roscoe & $47 \pm 1.00$ & $0.05>P>0.02$ & $1.30 \pm 0.01$ & $0.5>P>0.1$ \\
\hline 30 & $(\mathrm{~V} / 29)$ & $\begin{array}{l}\text { Reference standard antidote } \\
\text { (standard antisera } \\
\text { /immunoglobulin's) }\end{array}$ & $31.5 \pm 0.50$ & $\begin{array}{l}\text { Select to } \\
\text { compare }\end{array}$ & $1.08 \pm 0.02$ & $\begin{array}{l}\text { Select to } \\
\text { compare }\end{array}$ \\
\hline
\end{tabular}

Generally, snake venom is an intricate mixture of various proteins (>90\%) and most of them are enzymes, particularly (40\%) phospholipases $\mathrm{A}_{2}$ [30]. Among different complications, hepatic injury is one of the deadly venomous effects produced by cobra bite [31, 32]. Naja naja karachiensis venom caused significant increase in ALT and AST levels (dose dependently) that are surrogate markers for liver toxicity either by direct action or immunological (hypersensitivity) reaction. The allergic reactions reactions are not dose dependent which clarify lethal effects to the cytoplasm and mitochondrial membranes of the hepatic cells [25, 33]. Phospholipases enzymes, abundant in Naja naja karachiensis venom, are responsible for breakdown of membranous phospholipids and therefore resulted in cellular injury along with inflammation [24]. $\mathrm{PLA}_{2}$ is attributed to decrease in $\mathrm{Na}^{+} / \mathrm{K}^{+}$ATPase activities and led to greater influx of sodium ions and water molecules into the cell. Subsequently, plasma membrane lipid bilayer disorganized and eventually resulted in hepatic cells death $[33,34]$. In addition, $\mathrm{PLA}_{2}$ is also found to cause anticoagulation in victims [6]. All pharmacological 
TABLE 5: Cardioprotective activity of medicinal plant extracts on LDH and CK-MB levels to neutralize snake bite envenomation in experimental rabbits.

\begin{tabular}{|c|c|c|c|c|c|c|}
\hline \multirow{2}{*}{\multicolumn{2}{|c|}{ Sr. number $\begin{array}{l}\text { Group V } \\
\text { (subgroup) }\end{array}$}} & \multirow{2}{*}{ Name of evaluated sample } & \multicolumn{2}{|c|}{$\mathrm{LDH}$} & \multicolumn{2}{|c|}{ CK-MB } \\
\hline & & & $\begin{array}{l}\mathrm{U} / \mathrm{L}(\text { mean } \pm \\
\text { SEM })\end{array}$ & $\begin{array}{l}P \text { value/ } \\
\text { comment }\end{array}$ & $\begin{array}{c}\mathrm{U} / \mathrm{L} \\
(\mathrm{mean} \pm \mathrm{SEM})\end{array}$ & $\begin{array}{l}P \text { value/ } \\
\text { comment }\end{array}$ \\
\hline 1 & $(\mathrm{~V} / 1)$ & Albizia lebbeck (L.) Benth. & $1357 \pm 1.00$ & $0.01>P>0.001$ & $4.1 \pm 0.85$ & $0.5>P>0.1$ \\
\hline 2 & $(\mathrm{~V} / 2)$ & Allium cepa $\mathrm{L}$. & $934 \pm 13.03$ & $0.1>P>0.05$ & $14.8 \pm 1.65$ & $P>0.5$ \\
\hline 3 & $(\mathrm{~V} / 3)$ & Allium sativum $\mathrm{L}$. & $1177 \pm 20.56$ & $0.05>P>0.02$ & $6.6 \pm 3.30$ & $0.5>P>0.1$ \\
\hline 4 & $(\mathrm{~V} / 4)$ & Althaea officinalisL. & $975.5 \pm 33.60$ & $0.5>P>0.1$ & $14.8 \pm 3.30$ & $P>0.5$ \\
\hline 5 & $(\mathrm{~V} / 5)$ & Bauhinia variegata $\mathrm{L}$. & $1972 \pm 3.00$ & $P>0.001$ & $8.3 \pm 6.76$ & $P>0.5$ \\
\hline 6 & $(\mathrm{~V} / 6)$ & Brassica nigra (L.) W. D. J. Koch & $855.5 \pm 0.50$ & $0.05>P>0.02$ & $9.0 \pm 0.85$ & $0.5>P>0.1$ \\
\hline 7 & $(\mathrm{~V} / 7 \mathrm{a})$ & $\begin{array}{l}\text { Calotropis procera (Aiton) W. T. } \\
\text { Aiton (exudates) }\end{array}$ & $1022 \pm 5.01$ & $0.02>P>0.01$ & $6.6 \pm 3.30$ & $0.5>P>0.1$ \\
\hline 8 & $(\mathrm{~V} / 7 \mathrm{~b})$ & $\begin{array}{l}\text { Calotropis procera (Aiton) W. T. } \\
\text { Aiton (flowers) }\end{array}$ & $1114 \pm 1.00$ & $0.02>P>0.01$ & $61.8 \pm 10.9$ & $0.5>P>0.1$ \\
\hline 9 & $(\mathrm{~V} / 8)$ & $\begin{array}{l}\text { Cedrus deodara (Roxb. ex D. } \\
\text { Don) G. Don }\end{array}$ & $1230 \pm 23.57$ & $0.05>P>0.02$ & $41.2 \pm 31.66$ & $P>0.5$ \\
\hline 10 & $(\mathrm{~V} / 9)$ & Citrus limon (L.) Burm. f. & $1831 \pm 65.69$ & $0.05>P>0.02$ & $73 \pm 14.1$ & $0.5>P>0.1$ \\
\hline 11 & $(\mathrm{~V} / 10)$ & Citrullus colocynthis (L.) Schrad & $827 \pm 6.51$ & $0.1>P>0.05$ & $05 \pm 1.66$ & $0.5>P>0.1$ \\
\hline 12 & $(\mathrm{~V} / 11)$ & Cuminum cyminum L. & $1589 \pm 22.56$ & $0.02>P>0.01$ & $5.8 \pm 0.80$ & $0.5>P>0.1$ \\
\hline 13 & $(\mathrm{~V} / 12)$ & $\begin{array}{l}\text { Enicostemma hyssopifolium } \\
\text { (Willd.) I. Verd. }\end{array}$ & $1615 \pm 1.51$ & $P>0.001$ & $9.85 \pm 1.66$ & $0.5>P>0.1$ \\
\hline 14 & $(\mathrm{~V} / 13)$ & Fagonia cretica L. & $1418 \pm 13.03$ & $0.02>P>0.01$ & $11 \pm 2.52$ & $P>0.5$ \\
\hline 15 & $(\mathrm{~V} / 14)$ & Leucas capitata Desf. & $783 \pm 10.02$ & $0.5>P>0.1$ & $14 \pm 0.80$ & $P>0.5$ \\
\hline 16 & $(\mathrm{~V} / 15)$ & Matthiola incana (L.) W. T. Aiton & $1428 \pm 6.51$ & $P>0.001$ & $08 \pm 1.66$ & $0.5>P>0.1$ \\
\hline 17 & $(\mathrm{~V} / 16)$ & Momordica charantia L. & $1475.5 \pm 3.51$ & $P>0.001$ & $15.6 \pm 2.45$ & $P>0.5$ \\
\hline 18 & $(\mathrm{~V} / 17)$ & Nerium indicum Mill & $1268 \pm 12.03$ & $0.02>P>0.01$ & $05 \pm 1.65$ & $0.5>P>0.1$ \\
\hline 19 & $(\mathrm{~V} / 18)$ & Ocimum sanctum $\mathrm{L}$. & $1335 \pm 12.03$ & $0.02>P>0.01$ & $12.3 \pm 2.45$ & $P>0.5$ \\
\hline 20 & $(\mathrm{~V} / 19)$ & Pinus roxburghii Sarg & $1050 \pm 1.00$ & $0.02>P>0.01$ & $08 \pm 1.65$ & $0.5>P>0.1$ \\
\hline 21 & $(\mathrm{~V} / 20)$ & Pistacia integerrima J. L. Stewart & $1135.5 \pm 0.5$ & $0.02>P>0.01$ & $13.1 \pm 1.65$ & $0.5>P>0.1$ \\
\hline 22 & $(\mathrm{~V} / 21)$ & Psoralea corylifolia $\mathrm{L}$. & $1153.5 \pm 0.5$ & $P>0.001$ & $17.3 \pm 2.50$ & $0.5>P>0.1$ \\
\hline 23 & $(\mathrm{~V} / 22)$ & Rhazya stricta Decne & $1538 \pm 20.05$ & $0.02>P>0.01$ & $4.1 \pm 0.85$ & $0.5>P>0.1$ \\
\hline 24 & $(\mathrm{~V} / 23)$ & Rubia cordifolia L. & $1078 \pm 16.04$ & $0.05>P>0.02$ & $6.6 \pm 0.00$ & $0.5>P>0.1$ \\
\hline 25 & $(\mathrm{~V} / 24)$ & Sapindus mukorossi Gaertn & $1460.5 \pm 5.51$ & $0.01>P>0.001$ & $4.1 \pm 0.80$ & $0.5>P>0.1$ \\
\hline 26 & $(\mathrm{~V} / 25)$ & Stenolobium stans (L.) Seem & $787 \pm 28.08$ & $P>0.5$ & $13 \pm 1.76$ & $P>0.5$ \\
\hline 27 & $(\mathrm{~V} / 26)$ & $\begin{array}{l}\text { Terminalia arjuna (Roxb. ex } \\
\text { DC.) Wight and Arn }\end{array}$ & $798.5 \pm 14.54$ & $0.5>P>0.1$ & $6.6 \pm 0.00$ & $0.5>P>0.1$ \\
\hline 28 & $(\mathrm{~V} / 27)$ & Trichodesma indicum (L.) Sm & $978.5 \pm 4.51$ & $0.05>P>0.02$ & $6.6 \pm 3.00$ & $0.5>P>0.1$ \\
\hline 29 & $(\mathrm{~V} / 28)$ & Zingiber officinale Roscoe & $888 \pm 2.00$ & $0.05>P>0.02$ & $17.3 \pm 0.80$ & $0.5>P>0.1$ \\
\hline 30 & $(\mathrm{~V} / 29)$ & $\begin{array}{l}\text { Reference standard antidote } \\
\text { (standard antisera } \\
\text { /immunoglobulin's) }\end{array}$ & $763 \pm 6.01$ & Select to compare & $09 \pm 0.85$ & Select to compare \\
\hline
\end{tabular}

effects are due to phospholipid hydrolysis or phospholipid competing biding mechanism with other coagulation factors, particularly FXa, or by both ways; however, it is very hard to pinpoint exact one [35]. Anticoagulant response posed by cobra $\mathrm{PLA}_{2}$ (anticoagulant enzyme) may aggravate hepatic injury as many anticoagulant agents cause liver damage that are idiosyncratic in nature [36].

Cardiac injury, particularly systolic heart arrest, is one of the well-known toxicities related to Naja naja subspecies [30]. In present study, Pakistani cobra venom was found to release two cystolic enzymes (LDH and CK-MB) that are sensitive indicators of myocyte injury [37]. It is due to the presence of myotoxic PLA $\mathrm{P}_{2}$ and other cardiotoxin(s) that are salient features of cobra venom that are responsible for cellular necrosis and cytotoxicity $[38,39]$. Cytotoxic effect of Naja naja karachiensis venom may not be overlooked even when antisera administration is late cause of toxic components to the microvasculature and thrombus produced which poses hindrance in access of immunoglobulins to the site of snake bite $[26,29,39]$. 


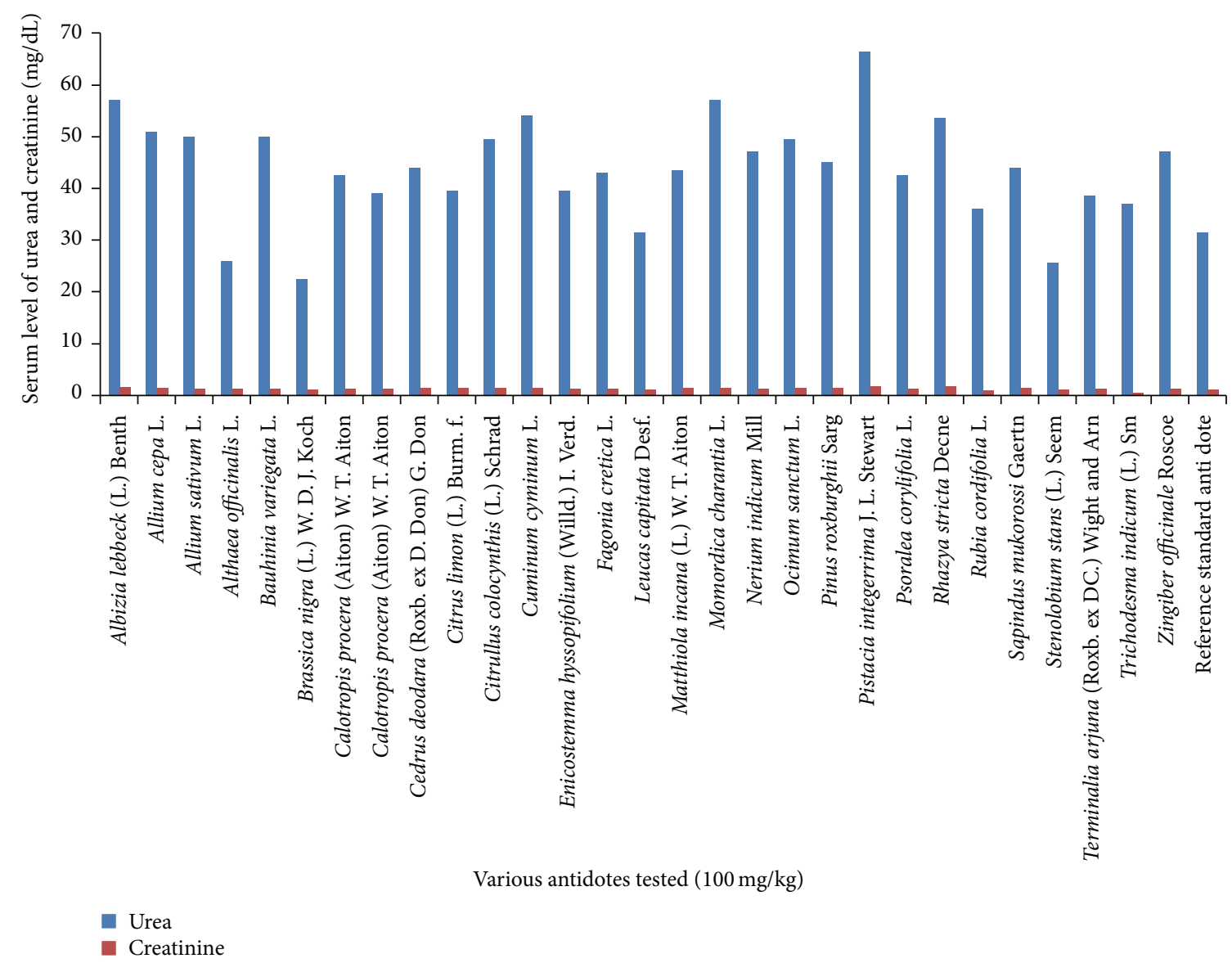

FIGURE 2: Comparison of various medicinal plant extracts with reference standard antivenom in neutralization of elevated serum urea and creatinine level posed by Naja naja karachiensis venom in rabbits.

Snake venom has been responsible for detrimental effects to the renal tissues [40]. Likewise, Naja naja krachiensis venom was found to cause severe renal damage by significant rise in serum urea and creatinine levels. Indeed, phospholipases enzymes are responsible for the increase in vascular permeability with hemorrhagic effects to the vital tissues in the victims. Subsequently, numerous lesions are produced related to the glomerular membrane and renal tubules either interstitial or vascular $[29,41,42]$. Presence of lymphocytes (white blood cells) and oedema in the cortical as well as medullary regions of renal tubules further confirmed the idea of renal damage (data has not shown) as reported previously with different snake venoms like Hemiscorpius lepturus [26, 43]. Apart of it, pharmacokinetic studies of Naja naja karachiensis venom with short lived radioisotope ${ }^{99 \mathrm{~m}} \mathrm{Tc}$ confirmed that kidneys and urinary bladder are the most saturated organs $(>70 \%)$ after intravenous injection in experimental rabbits (unpublished data by our group).

Medicinal plants of Pakistan are used to inhibit snake venom $\left(\mathrm{PLA}_{2}\right)$ enzymes [6]. Due to this effect, present study was designed to select twenty-eight medicinal plants of Pakistan to test their potentials as an antidote against toxicities produced by Naja naja karachiensis venom. It was fascinating that extract of Stenolobium stans (L.) Seem showed significant neutralization compared to that of reference standard (antisera) as reported previously by Asad et al., for inhibition of phospholipases $\mathrm{A}_{2}$ anticoagulant activity $[6,44]$. Present study indicated that extract of Stenolobium stans (L.) Seem possesses an endogenous inhibitor(s) to nullify venom $\left(\mathrm{PLA}_{2}\right)$ induced toxicities. Extracts of these plants material are routinely used in Pakistan by simple application in the form of paste to the affected area [45]. This practice provides effective first aid treatment as tiny molecules of an antidote diffuse favorably at the site of snake bite before hospitalization and abrogated the spreading of toxins [39]. It is owing to the various secondary metabolites like phenols, flavonoids, terpenoids, xanthenes, quinonoids, and so forth, as reported earlier to mask various enzymatic actions of cobra venom $[6,23]$. Indeed, secondary metabolites pose hindrance in binding of different snake venom enzymes to their potential targets; therefore, antidotal effect evoked. Other plants extract were not proved significantly effective to neutralize cobra venom, therefore cannot be declared useful plants in venom therapy. It is the need of the time to isolate lethal component(s) of cobra venom attributed to its major toxicities. Furthermore, characterization of antivenom 


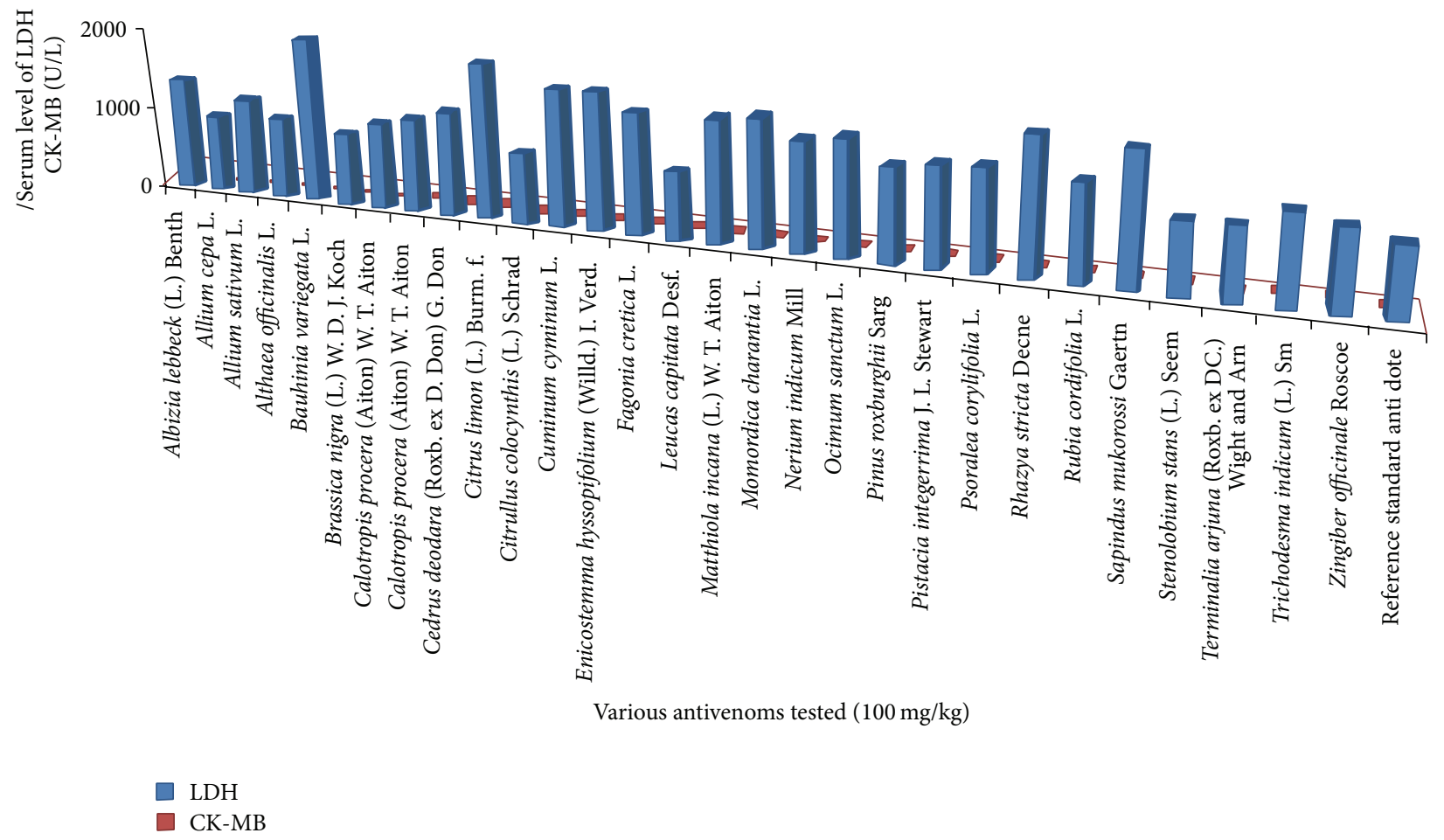

FIGURE 3: Comparison of various medicinal plant extracts with reference standard antisera in neutralization of LDH and CK-MB levels elevated by Naja naja karachiensis venom in rabbits.

compound(s) from medicinal plant extracts would be worth full for complete and effective treatment of snake bite in the future.

\section{Conflict of Interests}

The authors declare that there is no conflict of interests regarding the publication of this paper.

\section{Acknowledgments}

The authors are cordially thankful to Dr. Khan Muhammad Sajid (Deputy Chief Scientist MINAR) and Israr Ahmad (Radiopharmacist MINAR) for their valuable suggestions. Special thanks are due to Director COMSATS Institute of Information Technology Abbottabad Pakistan for the grant of study leave to conduct research in Multan Institute of Nuclear Medicine and Radiotherapy (MINAR), Nishtar Hospital, Multan, Pakistan.

\section{References}

[1] W. Wuster, "The cobras of the genus Naja in India," Hamadryad, vol. 23, pp. 15-32, 1998.

[2] P. E. P. Deraniyagala, "The taxonomy of the cobras of southeastern Asia, part 2," Spolia Zeylanica, vol. 29, pp. 205-232, 1961.

[3] T. M. Davidson, S. Schafer, and J. Killfoil, "Cobras," Wilderness and Environmental Medicine, vol. 6, no. 2, pp. 203-219, 1995.

[4] M. H. H. B. Asad, M. T. Razi, T. Khan et al., "Coagulopathies in Naja naja karachiensis (black Pakistan cobra) bites and its effect on coagulation tests upon storage of platelet poor plasma," Acta Poloniae Pharmaceutica, vol. 69, pp. 1031-1034, 2012.

[5] R. C. Hider, E. Karlsson, and S. Namiranian, "Separation and purification of toxins from snake venoms," in International Encyclopedia of Pharmacology and Therapeutics 11, A. L. Harvey, Ed., section 134-snake toxins, pp. 1-34, Pergamon Press, New York, NY, USA, 1991.

[6] M. H. H. B. Asad, M. T. Razi, Durr-e-Sabih et al., "Anti-venom potential of Pakistani medicinal plants: inhibition of anticoagulation activity of Naja naja karachiensis toxin," Current Science, vol. 105, pp. 1419-1424, 2013.

[7] D. Mebs, "Toxicity in animals. Trends in evolution?" Toxicon, vol. 39, no. 1, pp. 87-96, 2001.

[8] S. D. Aird, "Ophidian envenomation strategies and the role of purines," Toxicon, vol. 40, no. 4, pp. 335-393, 2002.

[9] T. Chijiwa, Y. Yamaguchi, T. Ogawa et al., "Interisland evolution of Trimeresurus flavoviridis venom phospholipase $\mathrm{A}_{2}$ isozymes," Journal of Molecular Evolution, vol. 56, no. 3, pp. 286-293, 2003.

[10] B. Lomonte, Y. Angulo, and L. Calderón, "An overview of lysine49 phospholipase A2 myotoxins from crotalid snake venoms and their structural determinants of myotoxic action," Toxicon, vol. 42, no. 8, pp. 885-901, 2003.

[11] B. L. Dhananjaya, B. S. Vishwanath, and C. J. M. D’Souza, "Snake venom nucleases, nucleotidases, and phosphomonoesterases," in Handbook of Venoms and Toxins of Reptiles, S. P. Mackessy, Ed., pp. 155-171, CRC Press, Boca Raton, Fla, USA, 2009.

[12] S. P. Mackessy, "Phosphodiesterases, ribonucleases and deoxyribonucleases," in Enzymes from Snake Venom, G. S. Bailey, Ed., pp. 361-404, Alaken, Fort Collins, Colo, USA, 1998.

[13] B. L. Dhananjaya, A. Nataraju, R. Rajesh et al., "Anticoagulant effect of Naja naja venom $5^{\circ}$ nucleotidase: demonstration 
through the use of novel specific inhibitor, vanillic acid," Toxicon, vol. 48, no. 4, pp. 411-421, 2006.

[14] B. L. Dhananjaya and C. J. M. D'Souza, “The Pharmacological role of phosphatases (acid and alkaline phosphomonoesterases) in snake venoms related to release of purines-a multitoxin," Basic and Clinical Pharmacology and Toxicology, vol. 108, no. 2, pp. 79-83, 2011.

[15] K. S. Girish, D. K. Jagadeesha, K. B. Rajeev, and K. Kemparaju, "Snake venom hyaluronidase: an evidence for isoforms and extracellular matrix degradation," Molecular and Cellular Biochemistry, vol. 240, no. 1-2, pp. 105-110, 2002.

[16] N. H. Tan and S. Y. Fung, "Snake venom L-amino acid oxidases," in Handbook of Venoms and Toxins of Reptiles, S. P. Mackessy, Ed., pp. 221-235, CRC Press, Boca Raton, Fla, USA, 2009.

[17] D. Mebs, Venomous and Poisonous Animals: A Handbook for Biologists, Toxicologists and Toxinologists, Physicians and Pharmacists, CRC Press, Boca Raton, Fla, USA, 2002.

[18] N. A. Abdoon and A. J. Fatani, "Correlation between blood pressure, cytokines and nitric oxide in conscious rabbits injected with Leiurus quinquestriatus quinquestriatus scorpion venom," Toxicon, vol. 54, no. 4, pp. 471-480, 2009.

[19] K. S. Latimer, E. A. Mahaffey, and K. W. Prasse, Duncan and Prasse's Veterinary Laboratory Medicine: Clinical Pathology, Wiley-Blackwell, New York, NY, USA, 5th edition, 2003.

[20] J. E. Harkness, P. V. Turner, S. V. Woude, and C. L. Wheler, Harkness and Wagner's Biology and Medicine of Rabbits and Rodents, Wiley-Blackwell, New York, NY, USA, 5th edition, 2010.

[21] F. S. Apple, "The specificity of biochemical markers of cardiac damage: a problem solved," Clinical Chemistry and Laboratory Medicine, vol. 37, no. 11-12, pp. 1085-1089, 1999.

[22] H. O. Alkadi, M. A. Noman, A. K. Al-Thobhani, F. S. Al-Meklafi, and Y. A. Raja'a, "Clinical and experimental evaluation of the effect of Khat-induced myocardial infarction," Saudi Medical Journal, vol. 23, no. 10, pp. 1195-1198, 2002.

[23] M. H. H. B. Asad, G. Murtaza, S. Siraj et al., "Enlisting the scientifically unnoticed medicinal plants of Pakistan as a source of novel therapeutic agents showing anti-venom activity," African Journal of Pharmacy and Pharmacology, vol. 5, no. 20, pp. 22922305, 2011.

[24] M. H. H. B. Asad, D. E. Sabih, B. A. Chaudhary et al., "Antihemolytic (anti-venom) activity of Pakistani medicinal plants upon Naja naja karachiensis venominduced hemolysis," Journal of Animal and Plant Sciences. In press.

[25] E. B. Ezenwanne and R. E. Ucheya, "A study of the serum concentrations of some hepatic enzymes in doses of aqueous leaf extract of Vernonia amygdalina in rabbits," International Journal of Animal and Veterinary Advances, vol. 4, pp. 80-83, 2012.

[26] Z. A. Mirakabadi, S. M. Khatoonabadi, S. H. Teimourzadeh, and G. H. H. Sabiri, "Serum enzymes studies in scorpion (Hemiscorpius lepturus) dose related envenomation in rabbits," Archives of Razi Institut, vol. 65, pp. 83-89, 2010.

[27] C. J. Green, J. Knight, S. Precious, and S. Simpkin, "Ketamine alone and combined with diazepam or xylazine in laboratory animals: a 10 year experience," Laboratory Animals, vol. 15, no. 2, pp. 163-170, 1981.

[28] S. S. Hasson, A. A. Al-Jabri, T. A. Sallam, M. S. Al-Balushi, and R. A. A. Mothana, "Antisnake venom activity of Hibiscus aethiopicus L. against Echis ocellatus and Naja n. nigricollis," Journal of Toxicology, vol. 2010, Article ID 837864, 8 pages, 2010.
[29] M. Tahir Razi, M. H. H. B. Asad, T. Khan et al., "Antihaemorrhagic potentials of Fagonia cretica against Naja naja karachiensis (black Pakistan cobra) venom," Natural Product Research, vol. 25, no. 20, pp. 1902-1907, 2011.

[30] M. Chethankumar and L. Srinivas, "Gangliosides as potential inhibitors of Naja naja venom PLA2 (NV-PLA2) induced human erythrocyte membrane damage," African Journal of Biochemistry Research, vol. 2, no. 1, pp. 8-14, 2008.

[31] T. R. Rahmy and K. Z. Hemmaid, "Histological and histochemical alterations in the liver following intramuscular injection with a sublethal dose of the Egyptian cobra venom," Journal of Natural Toxins, vol. 9, no. 1, pp. 21-32, 2000.

[32] B. Adzu, M. S. Abubakar, K. S. Izebe, D. D. Akumka, and K. S. Gamaniel, "Effect of Annona senegalensis rootbark extracts on Naja nigricotlis nigricotlis venom in rats," Journal of Ethnopharmacology, vol. 96, no. 3, pp. 507-513, 2005.

[33] M. A. Huffman, "Animal self-medication and ethno-medicine: exploration and exploitation of the medicinal properties of plants," Proceedings of the Nutrition Society, vol. 62, no. 2, pp. 371-381, 2003.

[34] B. W. Segelke, D. Nguyen, R. Chee, N. H. Xuong, and E. A. Dennis, "Structures of two novel crystal forms of Naja naja naja phospholipase $\mathrm{A}_{2}$ lacking $\mathrm{Ca}^{2+}$ reveal trimeric packing," Journal of Molecular Biology, vol. 279, no. 1, pp. 223-232, 1998.

[35] Q. Lu, J. M. Clemetson, and K. J. Clemetson, "Snake venoms and hemostasis," Journal of Thrombosis and Haemostasis, vol. 3, no. 8, pp. 1791-1799, 2005.

[36] S. Ehrenforth, J. F. Schenk, and I. Scharrer, "Liver damage induced by coumarin anticoagulants," Seminars in Thrombosis and Hemostasis, vol. 25, no. 1, pp. 79-83, 1999.

[37] M. Nandave, S. K. Ojha, S. Joshi, S. Kumari, and D. S. Arya, "Cardioprotective effect of Bacopa monneira against isoproterenol-induced myocardial necrosis in rats," International Journal of Pharmacology, vol. 3, no. 5, pp. 385-392, 2007.

[38] H. V. Patel, A. A. Vyas, K. A. Vyas et al., "Heparin and heparan sulfate bind to snake cardiotoxin: sulfated oligosaccharides as a potential target for cardiotoxin action," Journal of Biological Chemistry, vol. 272, no. 3, pp. 1484-1492, 1997.

[39] S. Yingprasertchai, S. Bunyasrisawat, and K. Ratanabanangkoon, "Hyaluronidase inhibitors (sodium cromoglycate and sodium auro-thiomalate) reduce the local tissue damage and prolong the survival time of mice injected with Naja kaouthia and Calloselasma rhodostoma venoms," Toxicon, vol. 42, no. 6 , pp. 635-646, 2003.

[40] M. Schneemann, R. Cathomas, S. T. Laidlaw, A. M. El Nahas, R. D. G. Theakston, and D. A. Warrell, "Life-threatening envenoming by the Saharan horned viper (Cerastes cerastes) causing micro-angiopathic haemolysis, coagulopathy and acute renal failure: clinical cases and review," QJM-Monthly Journal of the Association of Physicians, vol. 97, no. 11, pp. 717-727, 2004.

[41] J. Meier and K. Stocker, "Effects of snake venoms on hemostasis," Critical Reviews in Toxicology, vol. 21, no. 3, pp. 171-182, 1991.

[42] N. Marsh, D. Gattullo, P. Pagliaro, and G. Losano, “The gaboon viper, Bitis gabonica: hemorrhagic, metabolic, cardiovascular and clinical affects of the venom," Life Sciences, vol. 61, no. 8, pp. 763-769, 1997.

[43] M. H. Pipelzadeh, A. Jalali, M. Taraz, R. Pourabbas, and A. Zaremirakabadi, "An epidemiological and a clinical study on scorpionism by the Iranian scorpion Hemiscorpius lepturus," Toxicon, vol. 50, no. 7, pp. 984-992, 2007.

[44] M. H. H. B. Asad, Durr-e-Sabih, B. A. Choudary, A. F. Asad, G. Murtaza, and I. Hussain, "Compensatory effects of medicinal 
plants of Pakistan upon prolongation of coagulation assays induced by Naja naja karachiensis bite," Current Science, vol. 106, pp. 870-873, 2014.

[45] S. R. Baquar, Medicinal and Poisonous Plants of Pakistan, Printas Karachi Pakistan, 1st edition, 1989.

[46] I. Archetti, C. Tittarelli, M. Cerioli, R. Brivio, G. Grilli, and A. Lavazza, "Serum chemistry and hematology values in commercial rabbits, preliminary data from industrial farms in northern Italy," in Proceedings of the 9th World Rabbit Congress, pp. 11471152, Verona, Italy, 2008. 

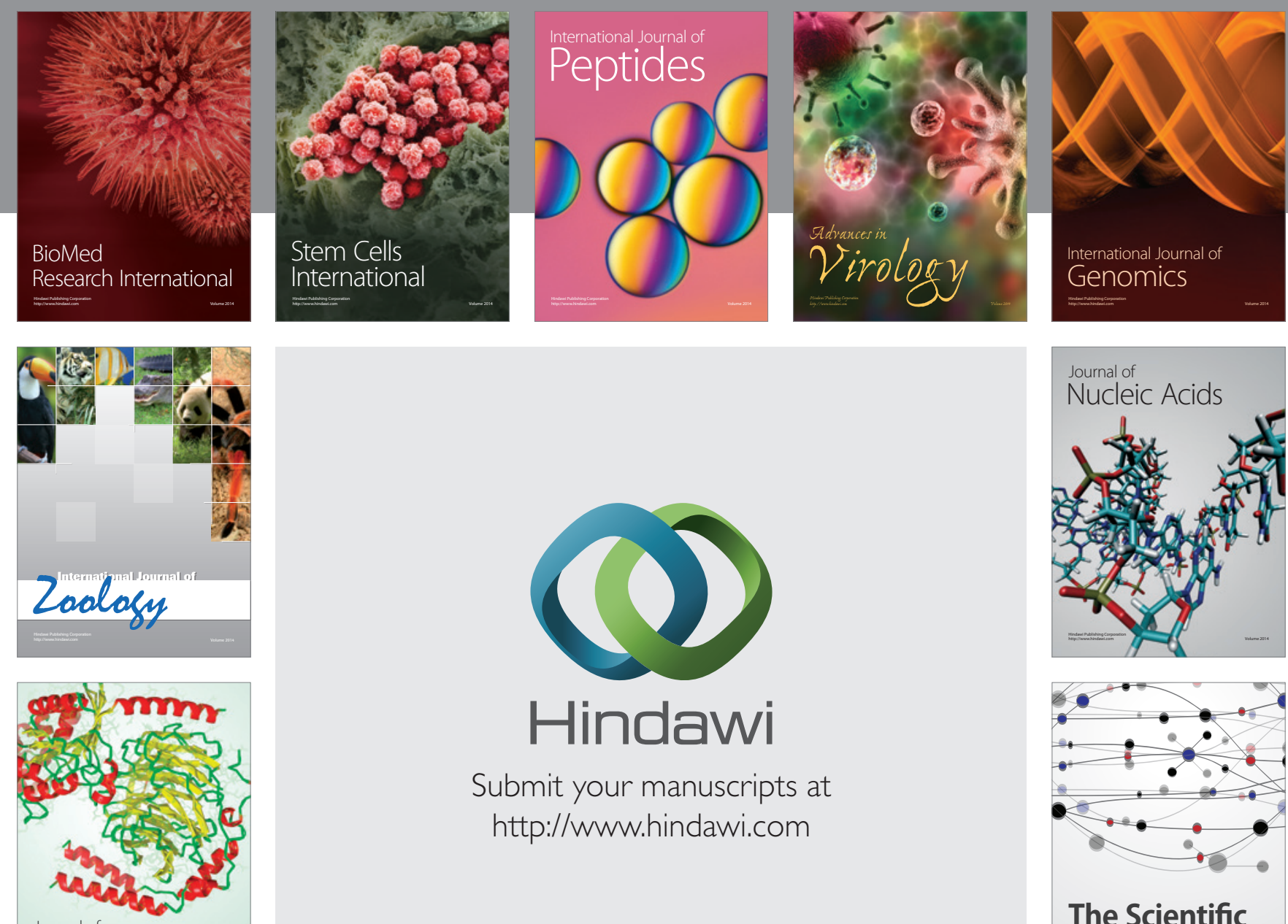

Submit your manuscripts at

http://www.hindawi.com

Journal of
Signal Transduction
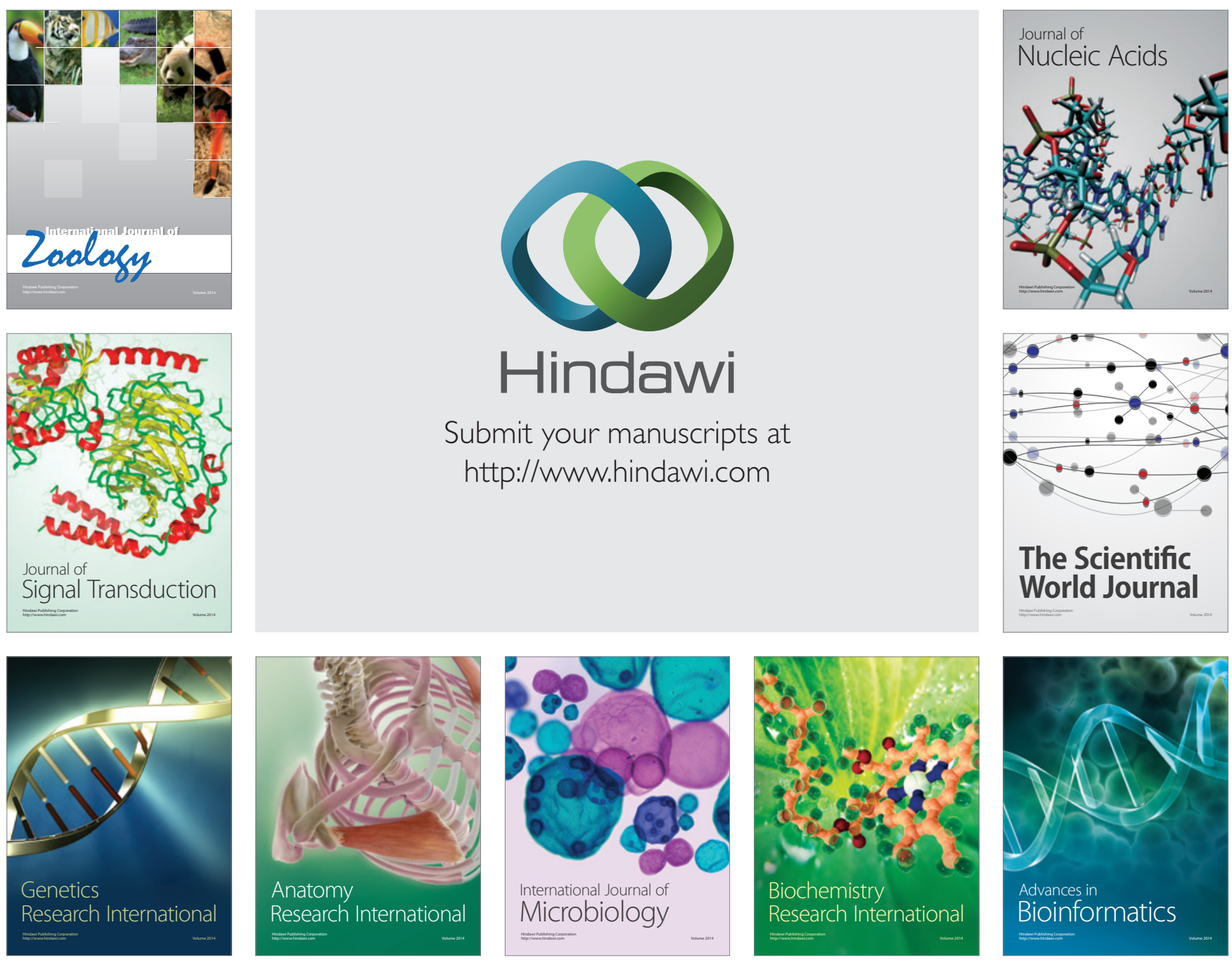

The Scientific World Journal
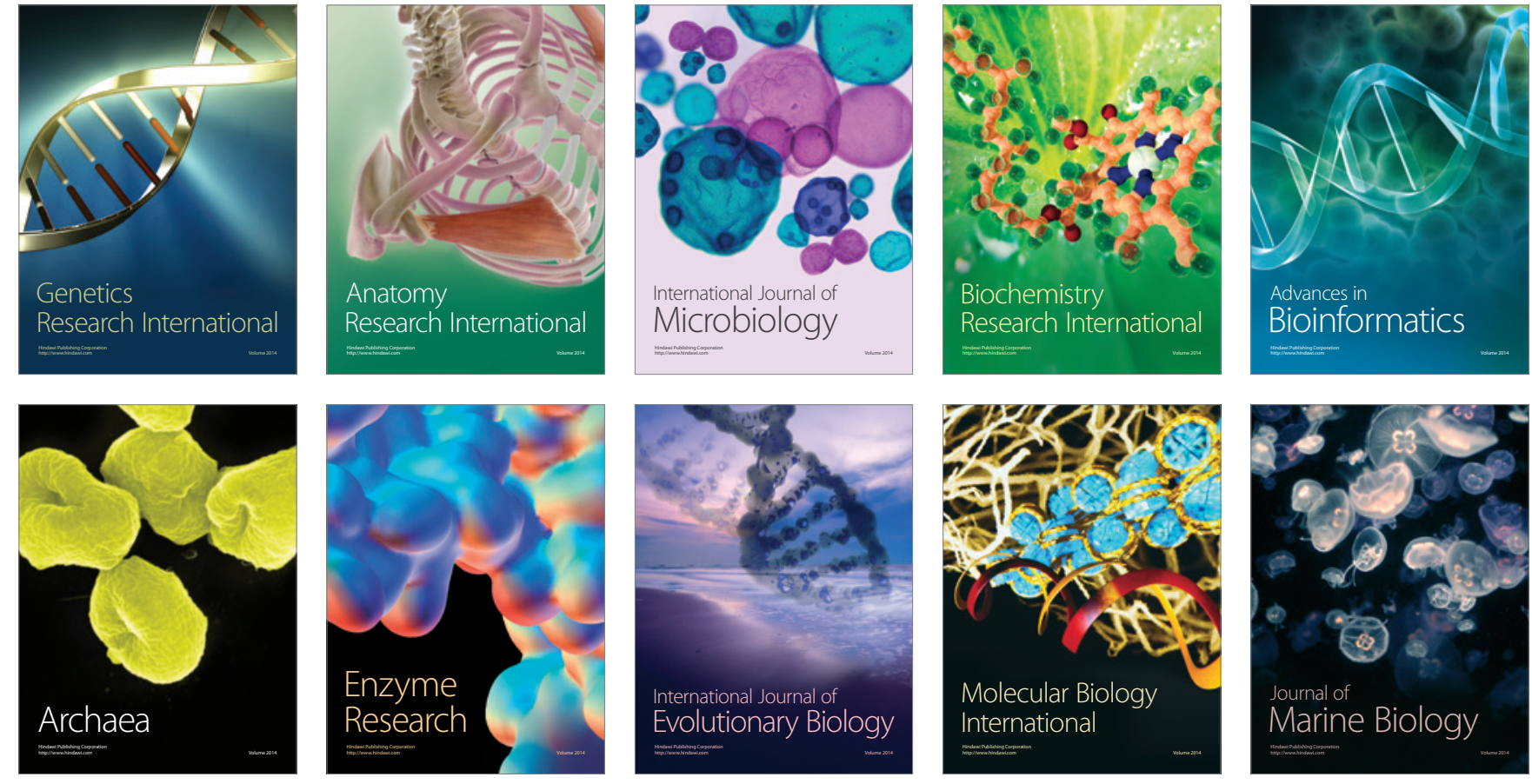